\title{
European Traditional Medicine - International Congress - Introductory Statement
}

\author{
Fabio Firenzuoli and Luigi Gori \\ Center of Natural Medicine, S. Giuseppe Hospital, Az USL 11, Empoli, Italy
}

\section{Why European Traditional Medicine?}

Traditional medicine (TM) is a large comprehensive term generally used to refer to systems such as traditional Chinese medicine, Indian ayurveda and Unani medicine, and to various forms of indigenous medicine, but also in Europe there is a very long history of traditional medicine that has a respectable historical and scientific dignity, and many European citizens still rely and trust in it for resolution of many minor and sometimes severe diseases. For all these reasons we decided to organize a congress based on the concept of European Traditional Medicine (ETM).

In Europe the dominant health care system is based on allopathic medicine, and also when TM has been partially or fully incorporated into the public health care system like in Tuscany, TM is still termed and considered "complementary", "alternative" or "non-conventional" medicine.

\section{Interconnections between Traditional and Conventional Medicines}

In Europe the pharmacopoeias of folk practitioners as well as professional medical health care providers contain thousands of medicines made from leaves, herbs, roots, bark, animal, mineral energetic substances and other materials found in nature like in Chinese, Ayurvedic, Unani and other systems; like many practices still based on energetic or traditional belief, so the aim of this congress is to explore and survey, very old and modern traditional based therapies and treatments curing the principles of scientific medicine (1). Discussions of the links between ETM and other traditional medicines therefore are mandatory, particularly when considering the importance of traditionally based therapies that are still a source of primary health care to about 70 percent of the world's population. Connections between traditional medicine and human health have been addressed and commented upon by many national and international political and sanitary bodies because: a) the good health of populations requires enlightened management of our social resources, economic relations, and of the natural world, and b) that many of today's public-health issues have their roots in lack of scientifically sustainable holistic approach to the patient c) many socioeconomic inequalities and irrational consumption patterns that jeopardize the future economic sustainability of health.

In the same context the conventional biomedical approach to health is based on methods of diagnosing and treating specific pathologies: one pathogen $=$ one disease, an approach that does not take into account connections between diseases, humanity, and some psychological aspects of suffering, and other socioeconomic factors such as poverty and education, and even the connections between disease and the environment in which sick people lives $(2,3)$.

Two examples of recently developed drugs, one from a plant and one from an animal, deserve special mention (4). The story of taxol and the Pacific yew (Taxus baccata) illustrates how we may be losing new medicines before species have been analyzed for their chemical content. The commercially useless Pacific yew was routinely discarded as a trash tree during logging of old growth forests in the Pacific northwest region of the United States or just used as a decorative plant in gardens until it was found to contain the compound taxol, a substance that kills cancer cells by a mechanism unlike that of other known chemotherapeutic agents: it prevents cell division by inhibiting the disassembly of the mitotic spindle. The discovery of the complex molecule taxol and its novel mechanism of action has led to the synthesis of several taxol-like compounds that are even 
more effective than the natural compound, which illustrates how a clue from nature can lead to the discovery of a new class of drugs that could have been extremely difficult to discover in the laboratory. Experimental and clinical studies have later shown that taxol may be one of the most promising new drugs available for the treatment of advanced carcinoma of the ovary, adjuvant treatment of node-positive breast cancer, non small-cell lung cancer, and second line treatment of AIDS related Kaposi's sarcoma.

The other example that deserves mention is the peptide compounds in the venom of cone snails, a genus of predatory snails numbering about 500 species that inhabits tropical coral reefs. The diversity of these compounds is so great that it may rival that of alkaloids in higher plants and secondary metabolites in microorganisms. Some of these peptide compounds, which have been shown to block a wide variety of ion channels, receptors and pumps in neuromuscular systems, have such selectivity that they are becoming important tools in neurophysiological research and of great value to clinical medicine. One voltage-sensitive calcium-channel blocker, $\omega$-conotoxin MVII-A, binds with great specificity to neuronal calcium channels, and from it has been developed ziconotide a new very potent analgesic substance non-opioid, non-NSAID, non-local anesthetic used for the amelioration of chronic pain.

\section{Connecting Traditional Medicine with Scientific Care}

Traditional medicine was the fundamental method used by humans to preserve their health and avoid diseases since the dawn of time. And it is not alternative or complementary for those who live far from medical facilities, in places where there are no physicians, or where physicians and drugs are too expensive. And even where there are good physicians and therapies are inexpensive, people still take the lead in their own health care. And their search for alternative medicine and therapies can also be defined as the continuity of traditions, religious beliefs, and even quackery that nonspecialists practice in many ways to treat people, not rarely for severe diseases like cancer.

Ordinary people not rarely thinking to be provided with clear and simple information try to prevent and to treat many common health problems in their homes, which can stop diseases earlier and provide cheaper treatment; but this self treatment can give rise to side effects, drug interferences, uses of wrong substances, delay of diagnosis or treatments.

Complementary and traditional medicines are popular with patients, but physicians obviously do not feel comfortable with this situation because in the university students have been taught to give patients only scientifically proved substances and therapies and...primum non nocere. Their fear is based on the right perception that some TM practitioners have dubious qualifications and competence, and that too little is known about the efficacy and safety of many complementary therapies. It follows that research is needed not only in the interest of the patient, but also of the professional health caregiver, and the modern relationship between patient and medicine based on the concept of "cure and taking care". So the point is which type of research? Strictly based on the principles of mainstream medicines? Sometimes it is impossible. So is mandatory to deal with these scientific and methodological issues and problems to find the way to fully connect traditional medicine in mainstream medicine, remembering that as in all areas of science, there can be no short cut to rigorous research.

ETM 2007 - European Traditional Medicine - is the first international Congress to discuss together ethnomedicine of European Countries in search of clinical evidence. The quantity and quality of research in Traditional Medicines and Complementary and Alternative Medicines has largely increased over the last decades in parallel with the ever-spreading demand by patients; so is growing a need of expertise for evaluating this challenging field and to improve a correct relationship between patients and health caregivers. The ETM Congress can give you the opportunity to hear the latest results of leading researchers, representing a wide range of different therapies and study designs in the emerging field of Traditional Medicines. In addition to research data, new ideas on mechanisms, strategies, ethical issues and methodological approaches will be discussed in plenary lectures.

The Center of Natural Medicine of Empoli Hospital is proud to host this outstanding event. Many thanks to Prof. Edwin L. Cooper, Editor in chief of ECAM Journal, for his meaningful cooperation.

\section{References}

1. Halberstain RA. Medicinal plants: historical and cross-cultural usage patterns. Ann Epidemiol 2005;15:686-99.

2. Lawoko S. Factors influencing satisfaction and well-being among parents of congenital heart disease children: development of a conceptual model based on the literature review. Scand J Caring Sci 2007;21:106-17.

3. Raina P, O'Donnel M, Rosenbaum P, Brehaut J, Walter SD, et al. The health and well-being of caregivers of children with cerebral palsy. Pediatrics 2005;115:e626-36.

4. Alves RR, Rosa IM. Biodiversity, traditional medicine and public health: where do they meet? J Ethnobiol Ethnomedicine 2007;3:14. 


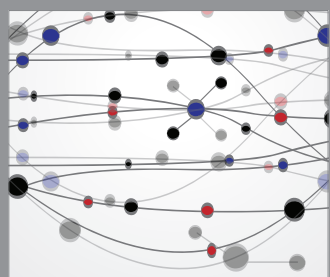

The Scientific World Journal
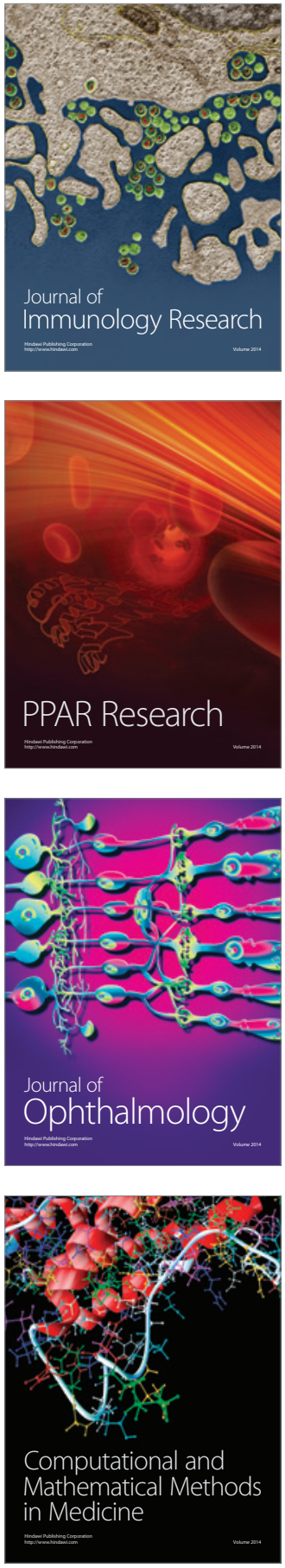

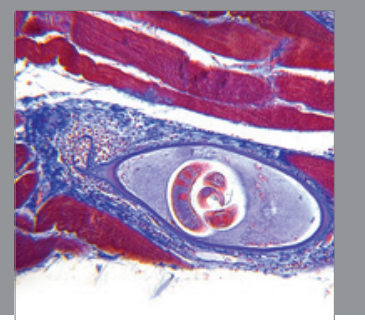

Gastroenterology

Research and Practice
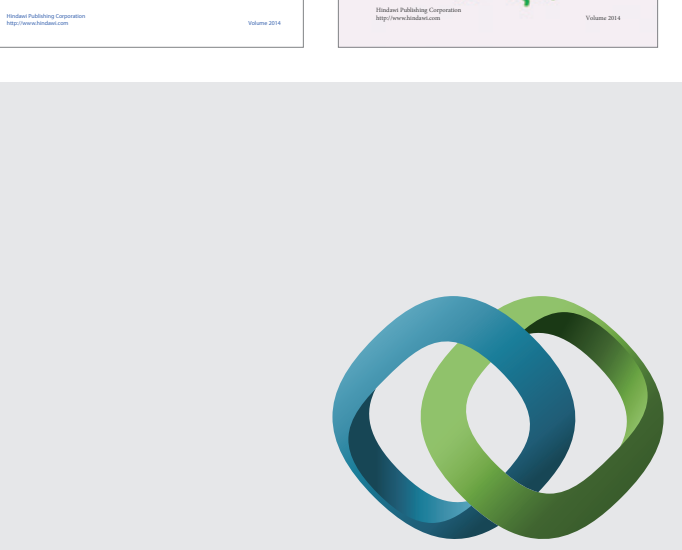

\section{Hindawi}

Submit your manuscripts at

http://www.hindawi.com
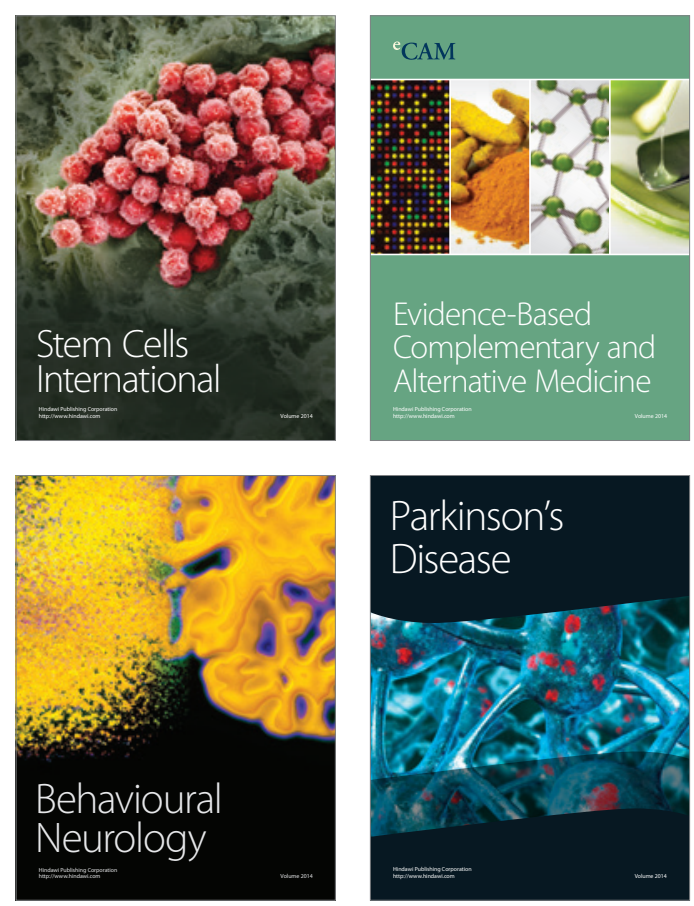

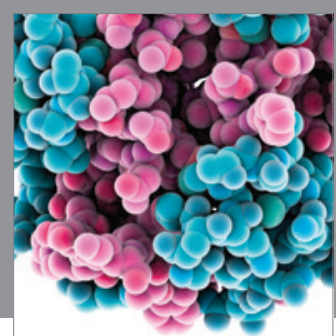

Journal of
Diabetes Research

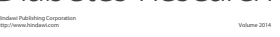

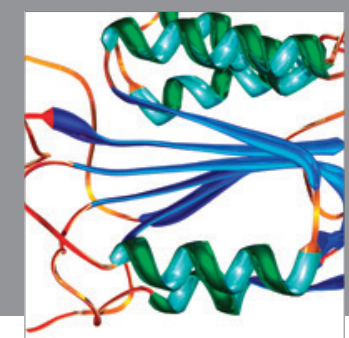

Disease Markers
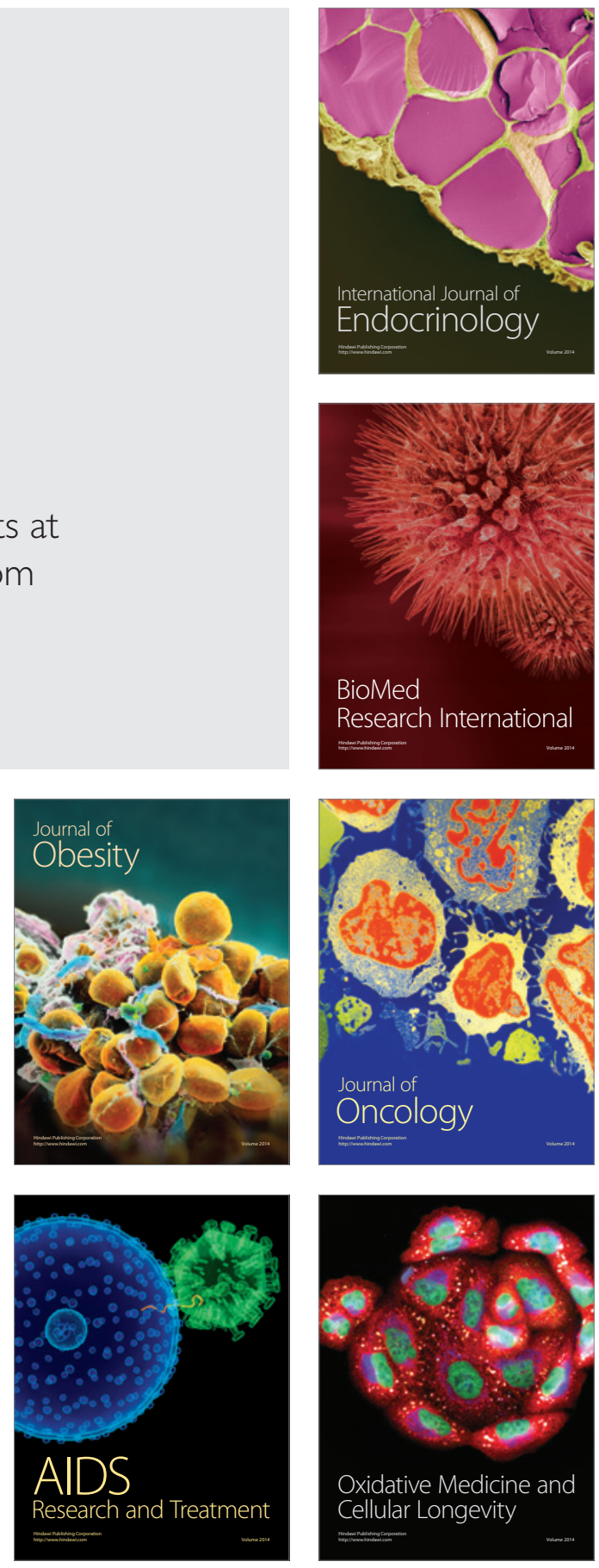